Rabaska

Revue d'ethnologie de l'Amérique française

FRASER, MARY L. Folklore of Nova Scotia. Introduction par IAN

BRODIE. Halifax, Formac, 2009 (1931), 115 p. ISBN

978-0-88780-861-6

\title{
Carmen d'Entremont
}

Volume 10, 2012

URI : https://id.erudit.org/iderudit/1013565ar

DOI : https://doi.org/10.7202/1013565ar

Aller au sommaire du numéro

Éditeur(s)

Société québécoise d'ethnologie

ISSN

1703-7433 (imprimé)

1916-7350 (numérique)

Découvrir la revue

Citer ce compte rendu

d'Entremont, C. (2012). Compte rendu de [FRASER, MARY L. Folklore of Nova Scotia. Introduction par IAN BRODIE. Halifax, Formac, 2009 (1931), 115 p. ISBN 978-0-88780-861-6]. Rabaska, 10, 255-258. https://doi.org/10.7202/1013565ar d'utilisation que vous pouvez consulter en ligne.

https://apropos.erudit.org/fr/usagers/politique-dutilisation/ 
de quelle façon le statut du chanteur québécois s'est modifié au cours des décennies. Également, on voit comment des artistes français, tels Maurice Chevalier ou Charles Trenet, ont influencé les Québécois dans leur pratique. On traite aussi des stratégies promotionnelles à la base de certains succès et échecs de chanteurs québécois en France. Finalement, l'auteur brosse un tableau des divers festivals et concours favorisant la diffusion de l'activité chansonnière de la Belle Province.

De Surmont détient un doctorat sur l'ingénierie lexicale comme solution à l'étude des phénomènes chansonniers. Il fait donc preuve d'un très grand souci de précision quant à l'usage des mots servant à décrire son objet d'étude. Dès le début du premier chapitre, il explique et motive les outils terminologiques qu'il emploie tout au long de l'essai. Un glossaire, qui gagnerait cependant à être plus complet, se trouve à la fin du livre. De plus, l'ouvrage contient des sources bibliographiques très exhaustives. Les notes en fin de chapitres sont abondantes, parfois celles-ci auraient pu être intégrées au corps du texte, ce qui aurait rendu la lecture plus agréable. Cela dit, ce livre se démarque par la qualité des théories qui y sont développées : nul doute, les outils méthodologiques définis par l'auteur favorisent l'avancement du discours théorique sur la chanson.

Louis-Martin SAVARD

Université de Moncton

Fraser, Mary L. Folklore of Nova Scotia. Introduction par Ian Brodie. Halifax, Formac, 2009 (1931), 115 p. ISBN 978-0-88780-861-6.

Folklore of Nova Scotia est la réédition d'un recueil paru antérieurement, en 1931. L'ouvrage est en fait une publication de la thèse de doctorat de l'auteur, Mary L. Fraser, une doctorante de l'Université Fordham, à NewYork, qui est originaire de Harbour Center, comté d'Antigonish. Fraser dresse ici un portrait général des croyances, des légendes et des coutumes des gens de la Nouvelle-Écosse. En plus d'avoir eu recours à diverses sources écrites, Fraser a aussi fait du terrain. Même si elle donne des informations provenant des peuples acadien et micmac, la majorité des faits et des anecdotes de son livre provient des Écossais situés, pour la plupart, dans les comtés d'Inverness et d'Antigonish, ce qui fait que le titre du recueil est trompeur et aurait pu s'intituler plus exactement : «Scottish Nova Scotian Folklore». Cette nouvelle édition comprend une introduction préparée par Ian Brodie, professeur associé de folklore à l'Université du Cap-Breton. En fait, cette introduction remplace celle de Fraser qui accompagnait la première édition, ce qui est fort dommage puisqu'on a l'impression qu'on nous a enlevé une 
partie importante du texte original. Brodie discute de l'origine du terme « folklore » ainsi que de l'intérêt pour l'étude de cette discipline. Il rappelle les tensions existant entre les professionnels et les amateurs de folklore dont les objectifs sont clairement différents. Il ne manque cependant pas de noter que les professionnels, c'est-à-dire ceux qui œuvrent dans des institutions universitaires, consultent souvent les collections des amateurs qui, selon lui, contiennent des informations utiles. Dans un sens, Brodie fixe à l'avance le ton du recueil qui contient des données intéressantes, mais qui est loin d'être exhaustif. Pour lui, le charme du recueil repose sur le fait que l'auteur perçoit les récits innovateurs du continent nord-américain comme étant tout aussi riches que ceux d'antan, contrairement aux folkloristes qui cherchaient infatigablement les récits les plus anciens et les plus «purs» (p. xi-xii). Puis Brodie présente l'auteur ainsi que les thèmes du livre.

L'ouvrage de Fraser se divise en seize chapitres couvrant un grand nombre de thèmes légendaires qui ont fait partie de la littérature orale des habitants des régions rurales de la Nouvelle-Écosse, au moins jusqu'au début du $\mathrm{Xx}^{\mathrm{e}}$ siècle. Le premier chapitre, «The Land and it's People », brosse brièvement l'histoire de cette province. L'auteur traite des premiers explorateurs et fait la liste des immigrants qui sont venus s'installer dans les diverses parties de la province. En plus de mentionner les nouveaux arrivants de la Nouvelle-Angleterre, la population allemande dans le comté de Lunenburg et l'établissement des Loyalistes, des Irlandais et des Écossais à travers la province, Fraser résume de façon sympathique l'histoire et surtout la déportation des Acadiens. Le deuxième chapitre, «Indian Myth and Legend », est surtout consacré au personnage mythique, Glooscap. Après avoir résumé la conversion des Micmacs au catholicisme, l'auteur signale quelques coutumes intéressantes. Elle note, par exemple, que l'on déposait des cadeaux, tels que des peaux de castor, des couteaux ou des flèches dans la fosse des morts (p. 15). Ensuite l'auteur donne plusieurs anecdotes entourant Glooscap. La majorité de ces dernières prennent la forme du conte étiologique : elles expliquent, entre autres, l'origine de la race micmaque ainsi que la provenance de certains traits géographiques, comme la formation d'îles et de roches. On dit, par exemple, que Glooscap aurait transformé en roches deux filles qui l'avaient regardé d'un air moqueur (p. 20). L'auteur en profite pour comparer les légendes micmaques à celles des Européens ; elle conclut que ces deux peuples étaient en communication, affirmation que Brodie juge peu vraisemblable (p. xiii). Le chapitre suivant, "Popular Superstitions », fournit une liste de remèdes et traite des signes qui apportent le malheur, comme le chat noir, ou la chance, comme l'écureuil (p. 29). Pour Fraser, « the superstitions of a race represent the religious beliefs of ancient peoples » (p. 24); toutefois, il est intéressant de noter qu'à part les pouvoirs attribués à l'eau bénite et aux médailles religieuses, la majorité des 
croyances relevées ici ne sont pas nécessairement basées sur une croyance religieuse. On dit, par exemple, qu' on pouvait faire disparaitre les verrues en jetant dans un puits un pois enveloppé d'une guenille (p. 25).

Les chapitres qui suivent sont, pour la plupart, organisés de la même façon; après avoir donné quelques lignes descriptives sur chaque thème, l'auteur énumère des croyances et des récits, surtout des anecdotes légendaires touchant majoritairement à des manifestations mystérieuses et surnaturelles. Les récits des chapitres « Second Sight », "Forerunners », "Apparitions due to Strong Wishing » et «The Return of the Dead» se penchent sur les présages, tels que les signes de mort, les apparitions et les revenants venus sur terre pour corriger une faute, remplir une promesse ou payer une dette. L'auteur raconte, entre autres, qu'un pêcheur noyé était revenu sur terre pour demander à un compagnon de régler la dette qu'il avait contractée auprès d'un magasin (p. 57-58). Le huitième chapitre porte sur les sorciers et la sorcellerie. On y apprend que l'on blâmait les sorciers lorsqu'une personne ou un animal tombait malade, et qu'une sorcière dans le comté d'Inverness faisait appel au diable si elle se voyait refuser quelque chose (p. 66). On trouve au neuvième chapitre, «Fairylore », des récits importés des Celtes portant sur les fées, ces petites créatures mystérieuses qui, comme le remarque Fraser, sont connues comme des lutins en Acadie. L'ouvrage comporte aussi quelques récits sur des trésors cachés (chapitre 10), sur des lieux hantés, des bruits et des apparitions étranges y compris un baril volant, un train mystérieux et un bateau fantôme (chapitre 11) ainsi que sur des sirènes (chapitre 12). Des anecdotes montrant la présence du diable dans la tradition orale des Écossais occupent le chapitre "The Devil in Folklore »; prenant tantôt la forme d'un animal, comme un chien ou un serpent, ou d'un étranger, il apparaissait surtout là où il y avait de l'alcool, de la danse et des jeux de cartes. Dans le chapitre «Legends Mainly Religious », l'auteur cite quelques croyances et anecdotes reliées aux fêtes calendaires ; elle mentionne, par exemple, la neige miraculeuse du mois de mai. Aussi, on y trouve deux contes étiologiques qui semblent avoir été placés un peu au hasard à la fin de la section ; le premier explique la disparition des porcs-épics et des mouffettes dans l'Île-du-Cap-Breton, et le deuxième, l'origine de la lisière noire sur le dos de l'aiglefin. Le quinzième chapitre, "Weather Lore », est réservé aux croyances météorologiques ; les Amérindiens, par exemple, savaient qu'une tempête s'en venait lorsqu'ils apercevaient un revenant en canot sur le lac Bras d'Or. Finalement, le dernier chapitre présente des coutumes reliées à la mort, au mariage et à la naissance.

Pour Ian Brodie, «Folklore of Nova Scotia is a flawed, wonderful book - or a wonderfully flawed book», autrement dit un livre imparfait sur le merveilleux ou un merveilleux livre imparfait. Le recueil de Fraser est un ouvrage de référence qui se lit bien, qui embrasse le contemporain et qui 
renseigne sur la richesse de l'imaginaire des Écossais de la Nouvelle-Écosse aux siècles derniers. Cependant, on peut lui reprocher quelques lacunes. En plus d'énumérer de nombreux thèmes légendaires, l'auteur aurait pu au moins développer une conclusion en analysant même brièvement ses découvertes. Aussi, comme le fait remarquer Ian Brodie, Fraser se montre inconstante dans l'usage de ses sources, les citations étant réduites au minimum et souvent incomplètes. Heureusement, Brodie a tenté de les rétablir en donnant des références plus complètes. Il avoue cependant qu'il s'est quelquefois basé sur des suppositions éclairées (p. xx). En outre, l'auteur a souvent omis le nom de plusieurs informateurs pour garder l'anonymat; et lorsque le nom est donné, le lecteur ne connaîtra ni son âge, ni son lieu d'origine, ni la date de la cueillette du récit, toutes des informations que le spécialiste aurait jugées essentielles. En dépit de ses lacunes, la nouvelle édition de ce recueil permet de faire connaître l'auteur, Mary Fraser, une religieuse qui a joué un rôle important dans la cueillette de matériel folklorique en Nouvelle-Écosse, et de l'ajouter à la liste des femmes collectrices au Canada.

Carmen d'Entremont

Université Sainte-Anne

Guay, Lorraine. Le Québec des îles. Québec, Les Presses de l'Université Laval, «Les régions du Québec », 2011, 192 p. ISBN : 978-2-7637-9230-9.

Ce petit livre est un ouvrage de vulgarisation dépouillé de l'érudition qui caractérise le précédent ouvrage du même auteur publié en 2003, dans le cadre du chantier des histoires régionales du Québec, intitulé À la découverte des îles du Saint-Laurent. De Cataracoui à Anticosti (Éditions du Septentrion, 2003, 400 p.). Ce titre aurait pu être appliqué au présent ouvrage de vulgarisation, vu que le sujet ne concerne pas autre chose que le Saint-Laurent et ses îles. Le Québec des îles surprend, au premier abord, mais peut se justifier par le fait que le dernier chapitre intitulé « Les habitants du Saint-Laurent » est le plus long des cinq qui composent le livre.

On y trouvera une foule de renseignements sur les îles continentales et maritimes ( $\mathrm{D}$ 'îles en archipels »), leurs définitions (« Des terres en mouvance »), les aspects mythiques et tragiques des îles ("Rêves et cauchemars »), les guerres et le commerce (« Des postes stratégiques ») et, pour finir, « Les habitants du Saint-Laurent $»$ où l'on trouvera quelques paragraphes relatifs à l'ethnographie. L'auteur s'appuie sur une bibliographie diversifiée et termine l'ouvrage par une énumération de repères chronologiques fort appréciés dans les livres d'histoire. Sauf l'emploi fautif du verbe essaimer, utilisé transitivement (p. 131), sans doute à cause d'une lacune dans la transcription du texte, et l'absence de la préposition par devant celui du bois (p. 122), le texte 